\section{Optimization Procedure for Planar Leaky-Wave Antennas with Flat-Topped Radiation Patterns}

\author{
Francesco Scattone, Mauro Ettorre, Senior Member, IEEE, \\ Ronan Sauleau, Senior Member, IEEE, Ngoc Tinh \\ Nguyen, Member, IEEE, \\ and Nelson J. G. Fonseca, Senior Member, IEEE
}

\begin{abstract}
We describe here an optimization procedure to shape the radiation pattern of planar 2-D leaky-wave antennas. The structures under investigation are superstrate configurations made by partially reflecting screens (impedances sheets) over a ground plane and excited by open ended waveguides. The antenna configuration providing the required radiation pattern is obtained by resorting to an ad-hoc optimization procedure that minimizes the mean squared error between the target pattern and the actual radiated far field. The radiated field is analytically evaluated using a Green's function spectral approach to speed up the optimization process. Several kinds of radiation patterns can be obtained using the proposed algorithm. In this work, we focus our attention on flat-topped radiation patterns, suitable as element pattern for phased array antennas covering a limited angular field-of-view such as those considered for space applications. The proposed procedure is validated by full-wave results and measurements.
\end{abstract}

Index Terms-Leaky-wave antennas, pattern shaping, phased array antennas.

\section{INTRODUCTION}

Leaky-wave antennas (LWAs) have been studied for decades mainly for their capability to enhance and/or modify the radiation properties of simple sources [1]. More recently, a particular class of planar 2-D LWAs consisting of an arrangement of dielectric substrates or impedance surfaces placed over a metallic ground plane (Fig 1), known as Fabry-Perot cavities or superstrate configurations, has received a great deal of attention. Such antennas are typically used to enhance the directivity of small sources in broadside direction [2]-[8] and to reduce the side lobe level (SLL) [9], [10]. Pattern shaping using planar LWAs has been also proposed in literature [11] [18]. The shaping is achieved by the tuning of the design parameters of the structures and, thus, the supported leakywave modes.

In this paper we propose an optimization procedure based on a Green's function spectral approach to tailor the radiation pattern using planar multi-layer LWAs in a superstrate configuration. The aim is a fast analysis and design tool providing the geometrical parameters of the structure radiating the required far field radiation pattern. The spectral transmission line

The authors would like to thank Brittany Region (ARED project 2012), ESA (co-sponsored PhD Ref. 267-2012) and ESF (NEWFOCUS Exchange Grant) for supporting the PhD activity.

F. Scattone, M. Ettorre, and R. Sauleau are with the Institut d'Electronique et de Télécommunications de Rennes (IETR), UMR CNRS 6164, University of Rennes 1, Rennes, France (e-mail: francesco.scattone@univ-rennes1.fr; mauro.ettorre@univ-rennes1.fr; ronan.sauleau@univ-rennes1.fr)

N. T. Nguyen is with LEAT, UMR CNRS 7248, University of Nice Sophia Antipolis, Sophia Antipolis, France (e-mail:ngoc-tinh.nguyen@unice.fr).

N. J. G. Fonseca is with Moltek Consultants Ltd for the European Space Agency, 2200 AG Noordwijk, The Netherlands (e-mail: nelson.fonseca@esa.int)

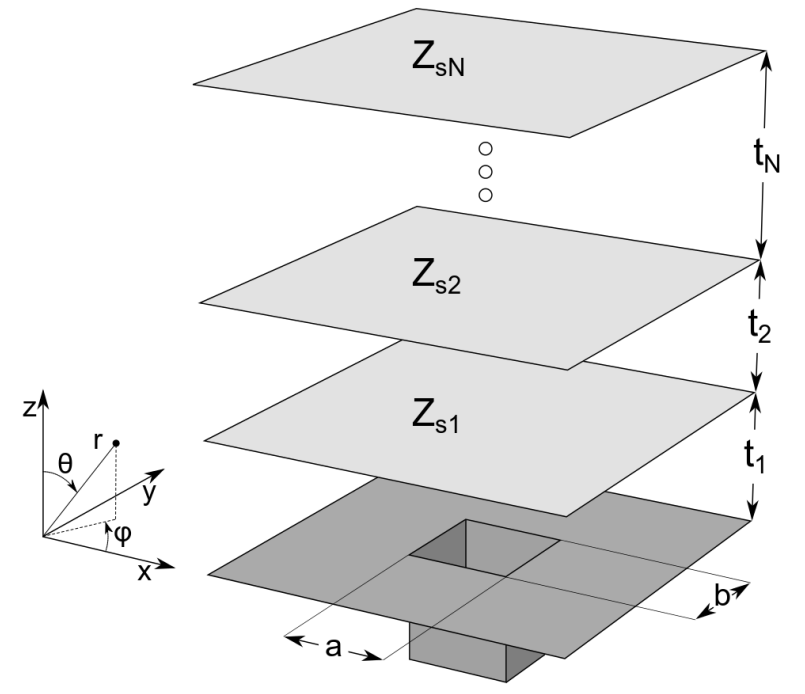

Fig. 1. Superstrate configuration consisting of $N$ impedance sheets above a ground plane. The source is a open ended waveguide with side dimensions $a$ and $b$.

representation of the superstrate configuration is adopted for finely tuning the design parameters of the antenna in order to minimize the mean squared error between the target radiation pattern and the actual radiated far field. The proposed method does not require the complete knowledge of the dispersion properties of the structure under investigation [16] or any bending or shaping of its physical profile [17]. Hereinafter, we focus our attention on the synthesis of flat-topped radiation patterns.

Flat-topped radiation patterns are generally adopted to provide uniform illumination over a defined angular region and low side lobe levels [19]. Phased array configurations of sources radiating flat-topped patterns are of interest for the reduction of the scan losses and side lobe levels, including grating lobes, over a defined field of view [10], [20]. These characteristics are suitable for on-board geosynchronous satellite applications, where a uniform illumination of the Earth and an efficient use of the available energy are required.

One of the first attempts to synthesize a flat-topped radiation pattern using a layered dielectric structure has been performed by Mailloux in [21]. In this work, a dielectric stratification was employed as a spatial filter in order to obtain a Chebyshevlike radiation pattern. Franchi and Mailloux followed a similar approach to obtain an angular filter using metallic grids [22] instead of dielectric slabs. In both cases, the design was performed using classical filter design techniques that did not take into account the actual nature of the sources used in the design. The idea was to apply the well-known formulas for Chebyshev band-pass filters, moving the problem from the frequency to the angular domain to derive the design parameters of the radiating structure. The solution proposed in [22] is the starting point of our optimization procedure for the flatted-topped case. However, as a difference with [22], the real spectral transmission line representation of the superstrate configuration is adopted for the evaluation of the radiated 


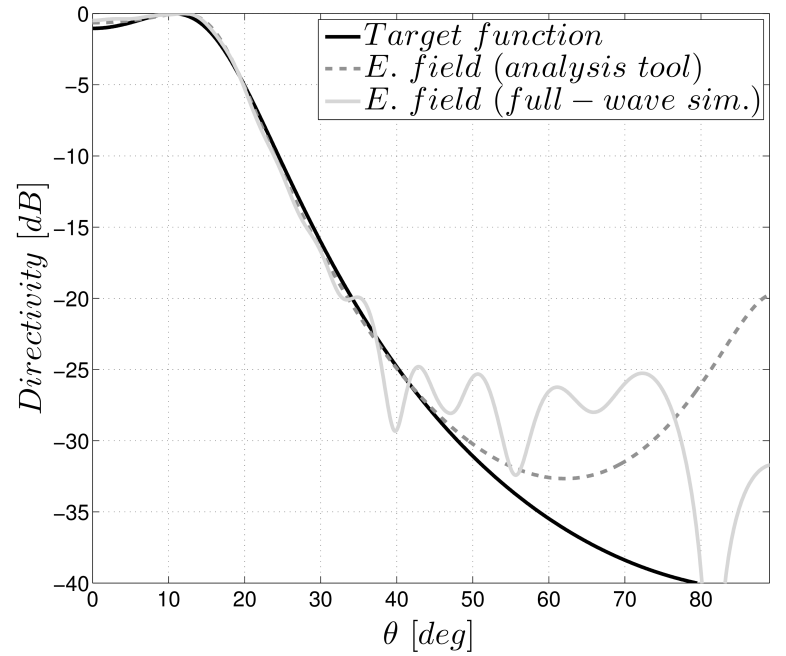

Fig. 2. Flat-topped radiation pattern in E-plane $\left(\phi=90^{\circ}\right)$. The black curve represents the analytical function to be approximated. The normalized farfield radiation pattern of the optimized structure evaluated using the in-house analysis tool and full-wave simulations are shown in dashed gray line and solid gray curve, respectively.

far field taking into account the position and nature of the feeding sources, leading to more accurate results and general procedure.

The paper is organized as follows. The optimization procedure for tailoring the radiation pattern is introduced in Section II. The results of the optimization procedure are shown in Section III for the case of flat-topped radiation patterns. Section IV presents a prototype operating at $10 \mathrm{GHz}$ and radiating a flat-topped radiation pattern. Full-wave and measurements results validate the synthesis procedure. The advantages of employing sources radiating a flat-topped pattern in a phased array configuration are highlighted in Section V. Finally, conclusions are drawn in Section VI.

\section{Optimization Procedure}

The structure under investigation consists of an arrangement of impedance surfaces over a metallic ground plane, as shown in Fig. 1. The antenna is fed by an open-ended waveguide with its aperture on the ground plane. The impedance surfaces can be synthesized using metallic gratings [4], [23], which are usually preferred to dielectric slabs for space applications. However, the proposed procedure can be used with any kind of partially reflecting screens.

The proposed procedure optimizes the design parameters of the antenna, namely: the surface impedances $Z_{s i}$, their spacings $t_{i}$ and the size of the feeding aperture $a, b$. The core of the optimization procedure is the minimization of the mean squared error (MSE) between the actual radiated electric field distribution and a target function:

$$
M S E=\int_{0}^{\theta_{c}}\left(\left|\mathbf{F}_{i}\left(\theta, \phi_{0}\right)\right|-\left|\mathbf{F}_{e}\left(\theta, \phi_{0}\right)\right|\right)^{2} d \theta,
$$

where $\theta_{c}$ represents the angular region under consideration. $\mathbf{F}_{e}\left(\theta, \phi_{0}\right)$ is the radiation pattern of the antenna under investi-

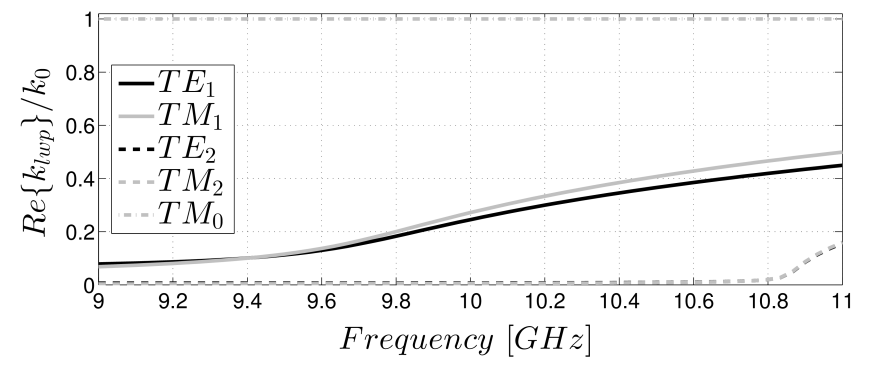

(a)

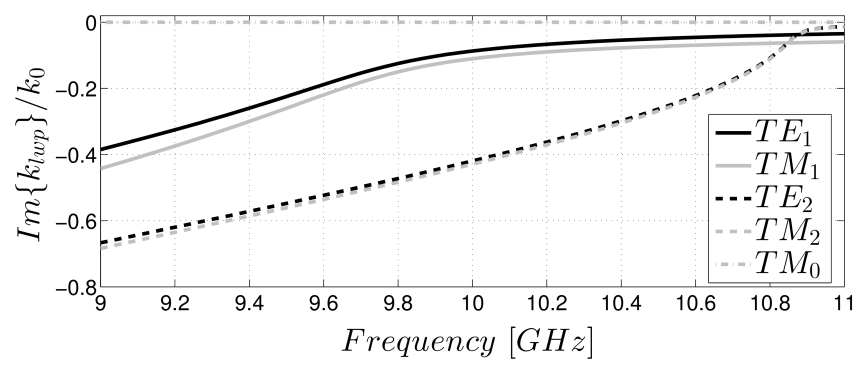

(b)

Fig. 3. Dispersion diagrams for the LWA generating a flat-topped radiation pattern. (a) Real part of the propagation constant expressed in radiation angles. (b) Normalized attenuation constant.

gation. It is analytically evaluated through the Green's function spectral approach used in [11], speeding up the computational time of the optimization tool. The target function $\mathbf{F}_{i}\left(\theta, \phi_{0}\right)$ is a mathematical function in the angular domain representing the ideal radiation pattern.

During the optimization, the parameters of the structure are varied in order to find a minimum of the MSE. This operation is performed with the aid of the constrained nonlinear optimization function included in the MATLAB optimization toolbox. An initial guess is needed to start the nonlinear optimization. In the present work, the initial guess is found by treating the radiation pattern as the frequency response of a Chebyshev bandpass filter in the angular domain, as in [22]. At each iteration, the radiation pattern of the resulting antenna is evaluated and compared with the target function. The process ends when a minimum of the MSE is found.

It is worth noting that the number of impedance sheets $N$ could be introduced as a variable in the optimization process. However, since it affects both the bandwidth [9] and the complexity of the antenna, it is convenient to define it a priori based on the requirement of the particular application.

\section{FLAT-TOPPED RADIATING STRUCTURE}

The optimization procedure proposed in Section II is used here to design a flat-topped radiation pattern, suited as element pattern of a phased array steering its main beam in a given field of view. This is particularly useful in arrays with a periodicity larger than $\lambda$ affected by the appearance of grating lobes in the visible range [20].

In the present case, the angular range to be uniformly covered is $\theta_{E}= \pm 8.6^{\circ}$, which corresponds to the visible surface of the Earth on geostationary satellites. The operating frequency is chosen equal to $f_{0}=10 \mathrm{GHz}$. The number of 


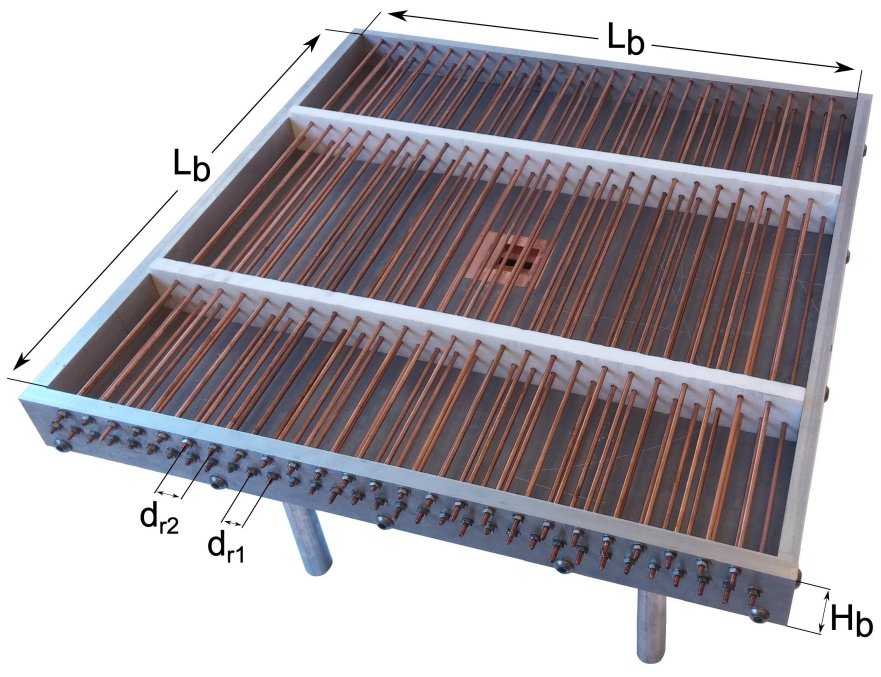

(a)

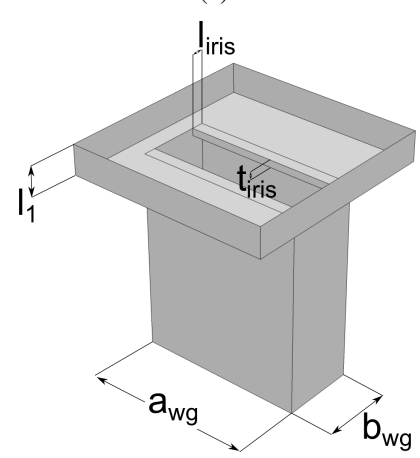

(b)

Fig. 4. (a) Realized prototype. (b) Pictorial view of the radiating aperture and corresponding matching network.

layers $N$ has been set equal to 2, allowing good performance over a fair bandwidth as well as a suitable roll-off. The latter determines the grating lobe rejection, once the feed is used in the array framework. Note that the following requirements has been chosen as an example and other specifications may be provided based on the considered application.

The target function $\mathbf{F}_{i}\left(\theta, \phi_{0}\right)$ to be approximated in the angular range up to $\theta_{c}=40^{\circ}$ by the radiation pattern, has been chosen equal to

$$
\mathbf{F}_{i}\left(\theta, \phi_{0}\right)=\frac{1}{1+A T_{2 N}\left(\frac{\sin \theta}{\sin \theta_{r}}\right)},
$$

with $T_{2 N}$ the Chebyshev polynomial of the first kind and order $2 N$. In the following, $N$ corresponds to the number of impedance sheets. The amplitude of the ripple of the target function is equal to $2 A$, while $\theta_{r}$ corresponds to half of the equal-ripple band edge.

Here, we consider $N=2$, with $\theta_{r}=15^{\circ}$ and $2 A=0.12$, corresponding to a ripple of $\sim 1 \mathrm{~dB}$. The value of $\theta_{r}$, selected at the design frequency, has been chosen larger than the requested $\theta_{E}$ in order to account for the dispersive behavior of the structure and resulting beamwidth variation with frequency over a typical operating band of about $7 \%$. This is further

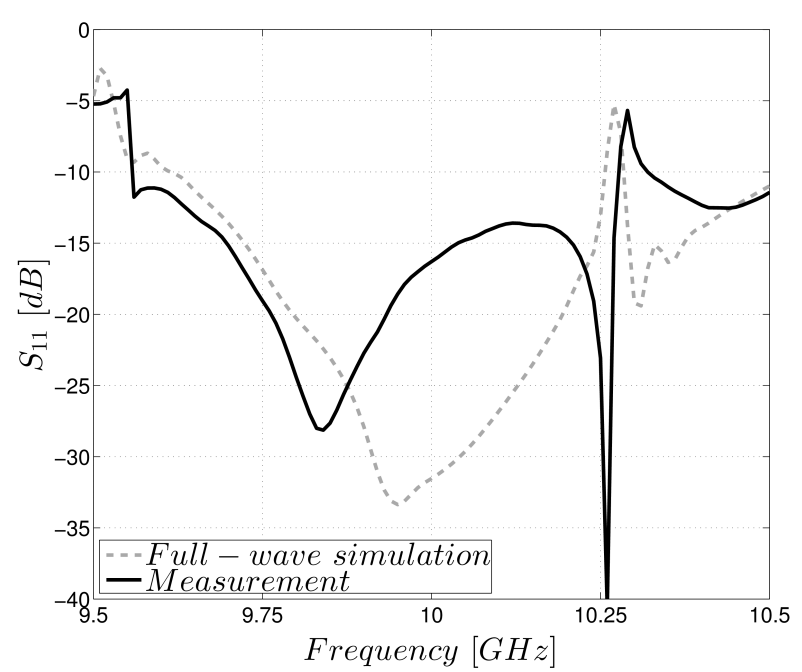

Fig. 5. Simulated and measured reflection coefficient.

discussed at the end of this section, based on the dispersion analysis results. Note that the optimization has been performed in E-plane $\left(\phi_{0}=90^{\circ}\right)$ of the LWA. This plane is the most critical one for superstrate configurations due to the presence of a leaky-wave mode radiating close to endfire [2]-[13], [15]. On the other hand, the azimuthal symmetry of the configuration will assure good radiation performance in all field cuts once the E-plane fulfills the imposed requirements on the radiation pattern [11], [13].

The computational time necessary to perform the whole optimization is less than two seconds on our workstation $(2 \times$ Intel Xeon E5-2650, 96 GB of RAM). The structure generated by the optimization process consists of two impedance sheets with $Z_{s 1}=j 34.8 \Omega, Z_{s 2}=j 115.2 \Omega$ separated by distances $t_{1}=t_{2}=13.52 \mathrm{~mm}$ (Fig. 1). The feed is a square waveguide [10]-[13] whose side has a length of $a=27 \mathrm{~mm}$. Fig. 2 shows the normalized electric field evaluated by the optimization tool and by the full-wave simulator ANSYS HFSS in E-plane $\left(\phi=90^{\circ}\right)$. A good agreement is observed between our tool and full-wave simulations. The simulation has been performed using two ideal impedance sheets. The lateral size of the antenna is equal to $30 \lambda_{0}$ (with $\lambda_{0}$ freespace wavelength) with negligible edge effects.

To gain a better understanding of the physical operation of the antenna, the dispersion diagrams for the proposed structure are illustrated in Fig. 3. These curves have been obtained by deriving the solutions of the dispersion equation associated to equivalent transmission line representation of the structure [11]. In particular, the real and imaginary part of the transverse propagation constant $k_{\rho}=\operatorname{Re}\left\{k_{l w p}\right\}-j \operatorname{Im}\left\{k_{l w p}\right\}$ are plotted. Several TE/TM leaky-wave modes are found. In particular, the couple of leaky-wave modes $\mathrm{TE}_{1} / \mathrm{TM}_{1}$ radiates close to $15^{\circ}$ at the design frequency $\left(\theta_{l w p} \approx \sin ^{-1}\left(\beta / k_{0}\right)\right)$ and is mainly responsible for the shape of the pattern. In fact, the couple $\mathrm{TE}_{2} / \mathrm{TM}_{2}$ is in cut-off at the central frequency, whereas the $\mathrm{TM}_{0}$ mode is radiating endfire in E-plane. Additionally, the variation of the radiating angle with frequency for the 


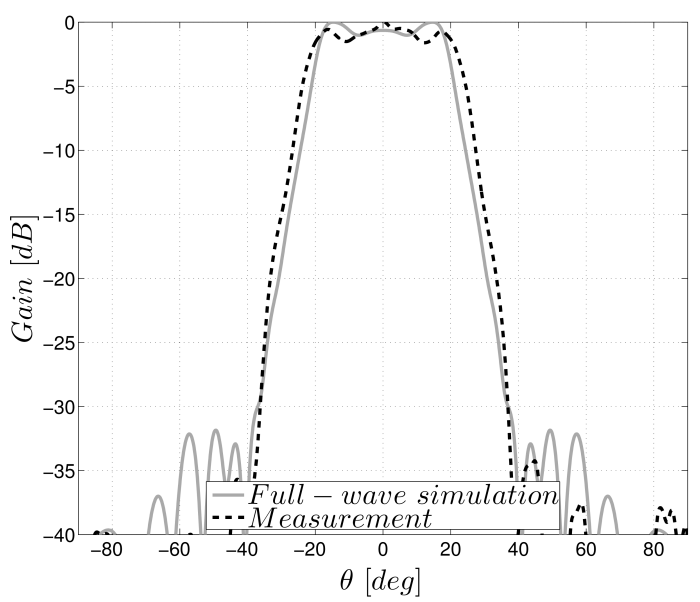

(a)

Fig. 6. Measured and simulated radiation patterns at the design frequency. (a) E-plane $\left(\phi=90^{\circ}\right)$. (b) $\mathrm{H}$-plane $\left(\phi=0^{\circ}\right)$.

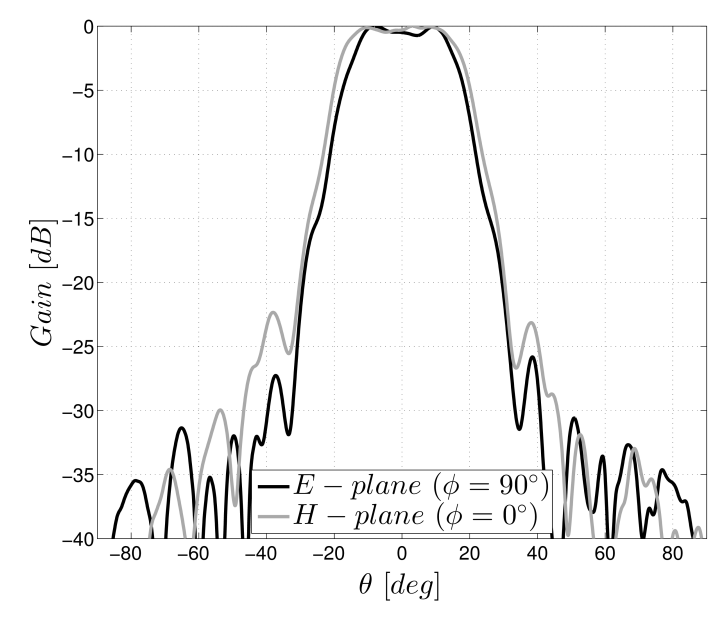

(a)

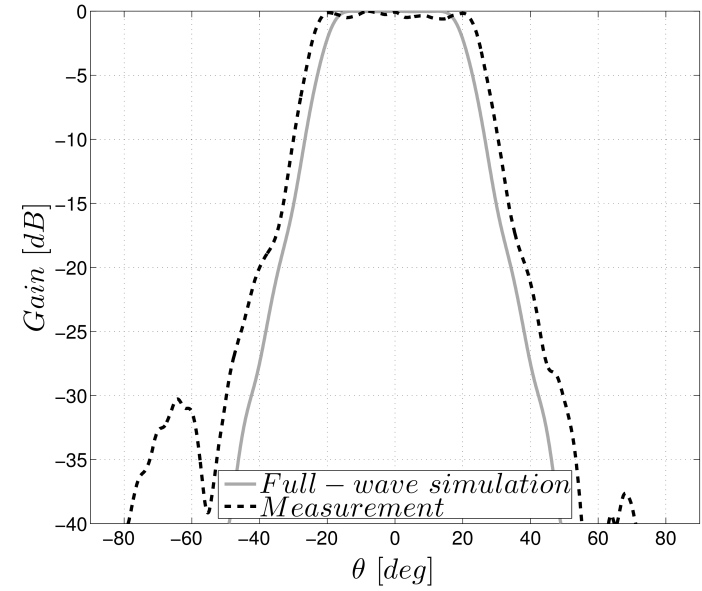

(b)

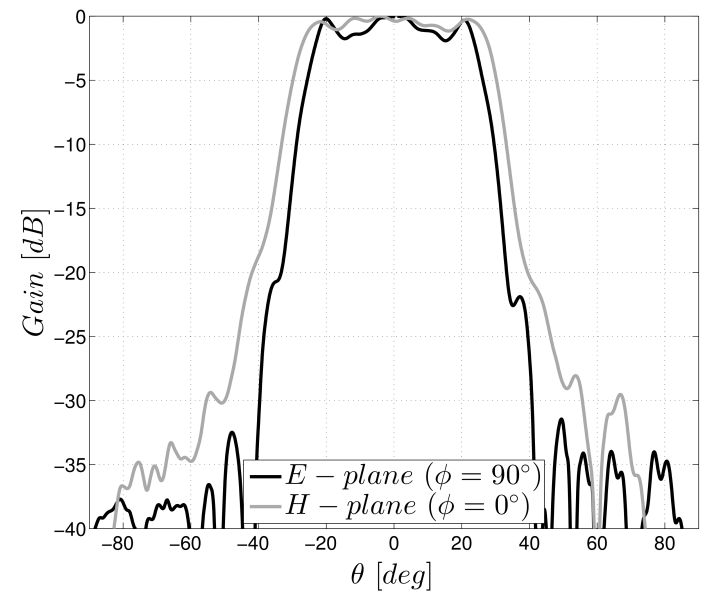

(b)

Fig. 7. Measured radiation patterns at the edges of the band of operation. (a) $9.6 \mathrm{GHz}$. (b) $10.2 \mathrm{GHz}$.

modes $\mathrm{TE}_{1} / \mathrm{TM}_{1}$, as reported in Fig. 3(a), clearly indicates that the lower operating frequencies are driving the minimum achievable beamwidth. Oversizing the field-of-view at the design frequency enables to remain compliant with a uniform coverage over the Earth at the lowest operating frequencies, around $9.6 \mathrm{GHz}$ for the proposed design.

\section{Prototype And Measurements}

The flat-topped antenna designed in the previous section has been manufactured and tested at IETR. The prototype is shown in Fig. 4. The impedance sheets have been synthesized using a periodic arrangement of metallic rods. HFSS fullwave simulations have been used to retrieve the required value of reactance for each layer. The metallic rods are made of copper with a diameter equal to $3 \mathrm{~mm}$. The final periodicity for the required impedance sheets are $d_{r 1}=12.25 \mathrm{~mm}$ and $d_{r 2}=15.48 \mathrm{~mm}$ for the first and the second layer, respectively. The behavior of these impedance layers is therefore inherently dispersive. In particular, the reactance of the sheet impedance varies by less than $10 \%$ (with respect to the nominal value at the operating frequency) within the considered band. Such a variation does not affect the overall performance of the antenna.

A metallic box has been placed around the antenna to supports the rods. The height of the box is $H_{b}=31 \mathrm{~mm}$ and the lateral size is $L_{b}=408 \mathrm{~mm}$. The latter has been chosen through a parametric analysis in such a way to minimize the SLL in E-plane. A metallic box has been placed around the antenna to support the rods. Two bars of Rohacell (relative permittivity $\epsilon_{r}=1.067$ ) have been placed within the metallic box of the final prototype to reduce the bending of the rods.

Fig. 4(b) shows a pictorial view of the feeding structure. The square waveguide used as source is connected to a standard WR-90 waveguide through a matching iris. The thickness and length of the iris are $t_{\text {iris }}=1 \mathrm{~mm}$ and $l_{\text {iris }}=1.54 \mathrm{~mm}$, respectively. The height of the square waveguide section 


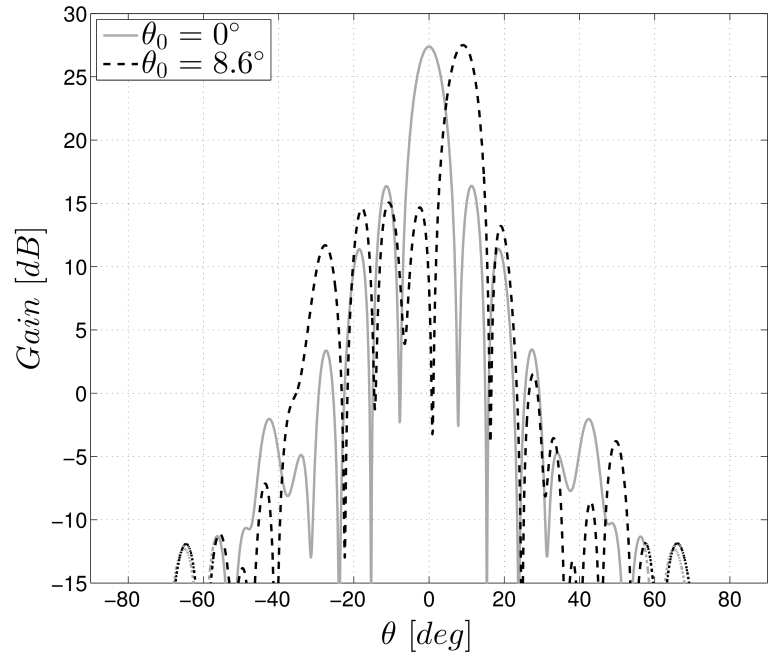

(a)

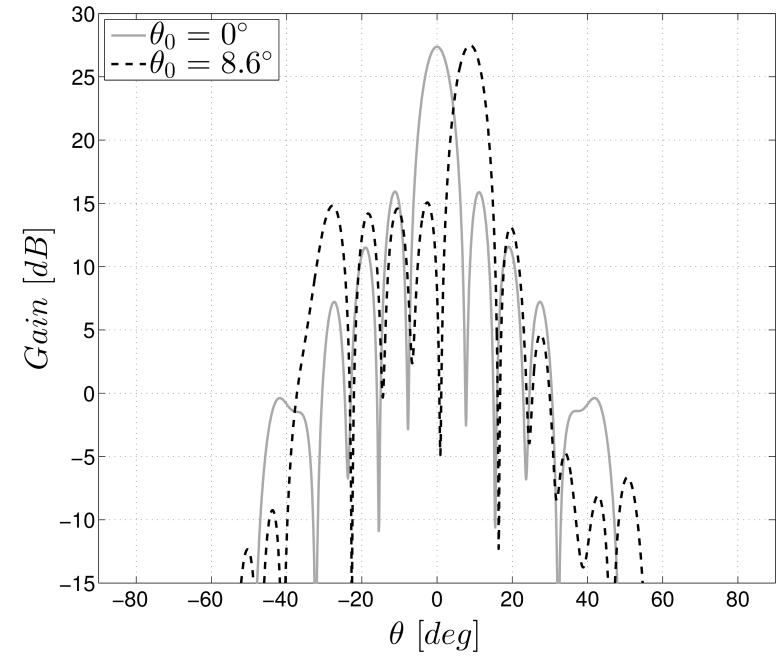

(b)

Fig. 8. Simulated radiation patterns of the $5 \times 5$ LWAs array pointing at broadside $\left(\theta_{0}=0^{\circ}\right)$ or at $\theta_{0}=8.6^{\circ}$. (a) E-plane. (b) H-plane.

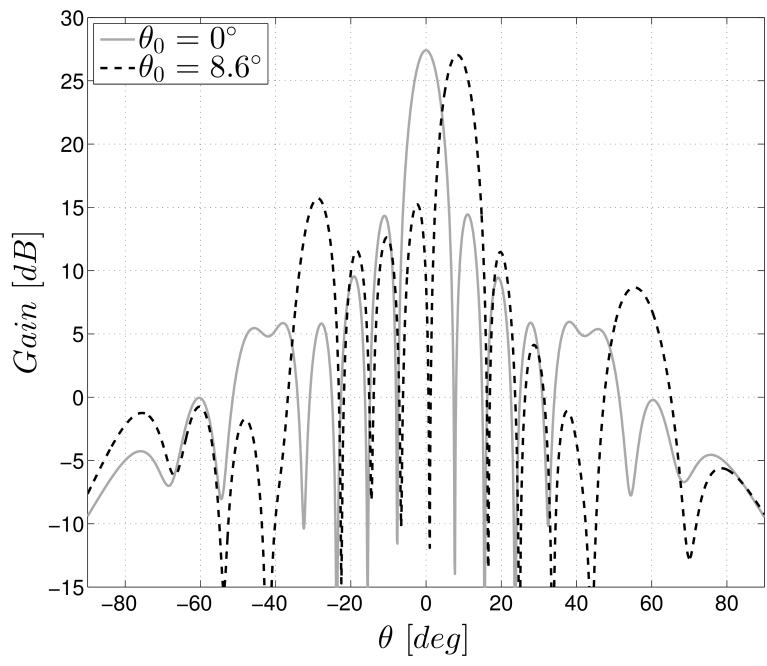

(a)

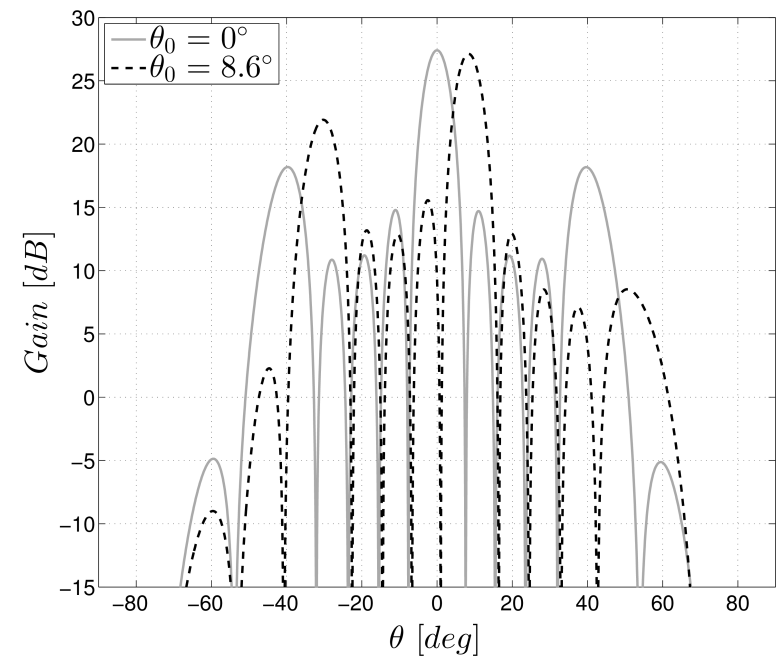

(b)

Fig. 9. Simulated radiation patterns of the $5 \times 5$ array bare configuration pointing at broadside $\left(\theta_{0}=0^{\circ}\right)$ or at $\theta_{0}=8 \cdot 6^{\circ}$. (a) E-plane. (b) H-plane.

is $l_{1}=4.17 \mathrm{~mm}$. The simulated and measured reflection coefficients are shown in Fig. 5. In both cases, the reflection coefficient is lower than $-10 \mathrm{~dB}$ over the same $7.5 \%$ band. However, we observe some discrepancies between the two curves that can be attributed to manufacturing imperfections and residual bending of the copper rods. Nevertheless, the results are considered acceptable.

In Fig. 6 we compare the simulation results with the measurements at the design frequency. A good agreement is obtained between the curves. A flat-topped radiation pattern has been achieved with a good roll-off figure. Small asymmetries can be observed in the measured radiation patterns attributed to the residual bending of the copper rods. The measured gain is equal to $12.1 \mathrm{~dB}$ at broadside.

The measured radiation patterns at $9.6 \mathrm{GHz}$ and $10.2 \mathrm{GHz}$ are shown in Fig. 7. The flat-topped shape is preserved within the considered band, but the beamwidth increases with the frequency. The measured broadside gain is $14.2 \mathrm{~dB}$ and $11.5 \mathrm{~dB}$ at the lower and upper edges of the band, respectively. Side lobe levels and roll-off values maintain excellent performance within the band.

\section{ARRAY PERFORMANCE}

Finally, we simulated the radiation pattern of an array of $5 \times 5$ square waveguides $(a=27 \mathrm{~mm})$ arranged in a square lattice with periodicity $d=1.5 \lambda_{0}=45 \mathrm{~mm}$ in the superstrate configuration analyzed in the previous sections. The outer dimensions of the antenna are the same as the one in Fig. 4(a). The array patterns for a beam pointing at broadside and at the edge of coverage $\left(\theta_{E}=8.6^{\circ}\right)$ are shown in Fig. 8. Limited 
scan losses are observed in the considered region. When scanning toward the edge of coverage, a grating lobe appears at $\theta_{G L}=\sin ^{-1}\left(\sin \theta_{0}-\lambda_{0} / d\right)=-31.1^{\circ}$, but its amplitude is below the side lobe level, by virtue of the synthesized pattern. In particular, the grating lobe levels are $-15.8 \mathrm{~dB}$ and $-12.7 \mathrm{~dB}$ for the E- and the H-planes, respectively.

As a benchmark solution, we took an array of $5 \times 5$ horn antennas without the superstrate and with the same periodicity $d=45 \mathrm{~mm}$ of the previous example. We refer to this configuration in the following as bare case. The side of the square apertures of each horn is $a=d=45 \mathrm{~mm}$, in order to maximize the aperture efficiency. The tapering length of the horns has been chosen equal to $108 \mathrm{~mm}$, maximizing the broadside gain for the given aperture [25]. Fig. 9 shows the array pattern in E- and H-planes. The antenna performance is degraded with respect to the superstrate configuration. In addition to the presence of scan losses on both planes, the grating lobe level is very high. Comparing the two solutions, the superstrate array provides a reduction of the grating lobes of $4.5 \mathrm{~dB}$ and $7.5 \mathrm{~dB}$ in E- and H-planes, with respect to the bare case. Even if not reported for brevity, similar performances have been obtained within the considered band of operation.

\section{CONCLUSION}

An optimization procedure to shape the radiation patterns of superstrate LWA antennas has been proposed. The considered LWA structures are based on impedance sheets above a ground plane. The optimization tool minimizes the mean squared error between the actual electric field radiated by the antenna and an ideal pattern. A spectral Green's function approach has been used to efficiently calculate the far-field radiated by the LWA structure at each step of the optimization procedure, speeding up the computational time. The final results of the optimization tool are the geometrical parameters of the considered configuration. An antenna radiating a flat-topped radiation pattern has been designed and prototyped, validating the synthesis procedure at $10 \mathrm{GHz}$. The same structure has been tested in array environment using numerical simulations. The flat-topped element pattern increases the rejection of grating lobes in array configurations with periodicities exceeding the operating wavelength and at the same time reduces the scan losses over a defined field of view.

\section{REFERENCES}

[1] R. E. Collin and F. J. Zucker, Antenna Theory, McGraw-Hill, New York, 1969.

[2] D. R. Jackson and A. A. Oliner, "A leaky-wave analysis of the high-gain printed antenna configuration," IEEE Trans. Antennas Propag., vol. 36, no. 7, pp. 905-910, Jul. 1988.

[3] D. R. Jackson, A. A. Oliner, and A. Ip, "Leaky-wave propagation and radiation for a narrow-beam multiple-layer dielectric structure," IEEE Trans. Antennas Propag., vol. 41, no. 3, pp. 344-348, Mar. 1993.

[4] T. Zhao, D. R. Jackson, J. T. Williams, H. D. Yang, and A. A Oliner, "2D periodic leaky-wave antennas-part I: metal patch Design," IEEE Trans. Antennas Propag., vol. 53, no. 11, pp. 3505-3514, Nov. 2005.

[5] T. Zhao, D. R. Jackson, and J. T. Williams, "2-D periodic leaky-wave antennas-part II: slot design," IEEE Trans. Antennas Propag., vol. 41, no. 11 , pp. $3515-3524$, Nov. 2005
[6] R. Sauleau, Ph. Coquet, D. Thouroude, J.-P. Daniel, and T. Matsui, "Radiation characteristics and performance of millimeter wave horn-fed gaussian beam antennas," IEEE Trans. Antennas Propag., vol. 51, no. 3, pp. 378-387, Mar. 2003.

[7] R. Sauleau, Ph. Coquet, T. Matsui, and J.-P. Daniel, "A new concept of focusing antennas using plane-parallel Fabry-Perot cavities with nonuniform mirrors," IEEE Trans. Antennas Propag., vol. 51, no. 11, pp. 3171-3175, Nov. 2003.

[8] G. Lovat, P. Burghignoli, and D.R. Jackson, "Fundamental properties and optimization of broadside radiation from uniform leaky-wave antennas," IEEE Trans. Antennas Propag., vol. 54, no. 5, pp. 1442-1452, May 2006.

[9] R. Gardelli, M. Albani, and F. Capolino, "Array thinning by using antennas in a Fabry-Perot cavity for gain enhancement," IEEE Trans. Antennas Propag., vol. 54, no. 7, pp. 1979-1990, Jul. 2006.

[10] D. Blanco, N. Llombart, and E. Rajo-Iglesias, "On the use of leaky wave phased arrays for the reduction of the grating lobe level," IEEE Trans. Antennas Propag., vol. 4, no. 4, pp. 1789-1795, Apr. 2014.

[11] A. Neto, N. Llombart, G. Gerini, M. D. Bonnedal, and P. De Maagt, "EBG enhanced feeds for the improvement of the aperture efficiency of reflector antennas," IEEE Trans. Antennas Propag., vol. 55, no. 8, pp. 2185-2193, Aug. 2007.

[12] N. Llombart, A. Neto, G. Gerini, D. M. Bonnedal, and P. De Maagt, "Leaky wave enhanced feed arrays for the improvement of the edge of coverage gain in multibeam reflector antennas," IEEE Trans. Antennas Propag., vol. 56, no. 5, pp. 1280-1291, May 2008.

[13] A. Neto, M. Ettorre, G. Gerini, and P. De Maagt, "Leaky wave enhanced feeds for multibeam reflectors to be used for Telecom satellite based links," IEEE Trans. Antennas Propag., vol. 60, no. 1, pp. 110-120, Sep. 2006.

[14] P. Burghignoli, F. Frezza, A. Galli, and G. Schettini, "Synthesis of broadbeam patterns through leaky-wave antennas with rectilinear geometry," IEEE Antennas Wirel. Propag. Lett., vol. 2, no. 1, pp. 136-139, 2003.

[15] F. Scattone, M. Ettorre, R. Sauleau, and N. J. G. Fonseca, "A flattopped leaky-wave source for phased arrays with reduced scan losses," Proceedings of the 8th European Conference on Antennas and Propagation (EuCAP), 2014, pp. 1220-1224, Apr. 2014.

[16] J.L. Gomez-Tornero, Adl.T. Martinez, D.C. Rebenaque, M. Gugliemi, and A. Alvarez-Melcon, "Design of tapered leaky-wave antennas in hybrid waveguide-planar technology for millimeter waveband applications," IEEE Trans. Antennas Propag., vol. 53, no. 8, pp. 2563-2577, Aug. 2005.

[17] I. Ohtera, "Diverging/focusing of electromagnetic waves by utilizing the curved leakywave structure: application to broad-beam antenna for radiating within specified wide-angle," IEEE Trans. Antennas Propag., vol. 47, no. 9, pp. 1470-1475, Sep. 1999

[18] E. Gandini, M. Ettorre, R. Sauleau, and A. Neto, "Mutual coupling reduction of Fabry-Perot SIW feeds using a double partially reflecting pin-made grid configuration," IEEE Antennas Wirel. Propag. Lett., vol. 10, pp. 647-650, 2011.

[19] N. T. Nguyen, R. Sauleau, and L. Le Coq, "Reduced-size double-shell lens antenna with flat-top radiation pattern for indoor communications at millimeter waves," IEEE Trans. Antennas Propag., vol. 59, no. 6, pp. 2424-2429, Jun. 2011

[20] R. J. Mailloux, "An overlapped subarray for limited scan application," IEEE Trans. Antennas Propag., vol. 22, no. 3, pp. 487-489, May 1974.

[21] R. J. Mailloux, "Synthesis of spatial filters with Chebyshev characteristics", IEEE Trans. Antennas Propag., vol. AP-24, no. 2, p. 174-181, Mar. 1976.

[22] P. Franchi and R. J. Mailloux, "Theoretical and experimental study of metal grid angular filters for sidelobe suppression," IEEE Trans. Antennas Propag., vol. 31, no. 3, pp. 445-450, May 1983.

[23] R. Sauleau, Ph. Coquet, and J.-P. Daniel, "Validity and accuracy of equivalent circuit models of passive inductive meshes. Definition of a novel model for 2D grids," International Journal of Infrared and Millimeter Waves, vol. 23, no. 3, pp. 475-498, Mar. 2002.

[24] R. F. Harrington, Time-harmonic electromagnetic fields, Wiley-IEEE Press, 2001.

[25] C. A. Balanis, Antenna Theory, Analysis and Design, Wiley, New York, 2012.

[26] G. L. Matthaei, E. M. T. Jones, and L. Young, Microwave filters, impedance-matching networks, and coupling structures, McGraw-Hill, New York, 1964 